\title{
The b-chromatic number of powers of cycles
}

\author{
Anja Kohl| \\ Faculty of Information Technology/Mathematics, HTW Dresden, Germany \\ received $16^{\text {th }}$ May 2011, revised $18^{\text {th }}$ July 2012, accepted $13^{\text {th }}$ March 2013.
}

\begin{abstract}
A $b$-coloring of a graph $G$ by $k$ colors is a proper vertex coloring such that each color class contains a color-dominating vertex, that is, a vertex having neighbors in all other $k-1$ color classes. The $b$-chromatic number $\chi_{b}(G)$ is the maximum integer $k$ for which $G$ has a $b$-coloring by $k$ colors. Let $C_{n}^{r}$ be the $r$ th power of a cycle of order $n$. In 2003, Effantin and Kheddouci established the $b$-chromatic number $\chi_{b}\left(C_{n}^{r}\right)$ for all values of $n$ and $r$, except for $2 r+3 \leq n \leq 3 r$. For the missing cases they presented the lower bound $L:=\min \left\{n-r-1, r+1+\left\lfloor\frac{n-r-1}{3}\right\rfloor\right\}$ and conjectured that $\chi_{b}\left(C_{n}^{r}\right)=L$. In this paper, we determine the exact value on $\chi_{b}\left(C_{n}^{r}\right)$ for the missing cases. It turns out that $\chi_{b}\left(C_{n}^{r}\right)>L$ for $2 r+3 \leq n \leq 2 r+3+\frac{r-6}{4}$.
\end{abstract}

Keywords: $b$-chromatic number, coloring, $b$-coloring, powers of cycles

\section{Introduction}

Let $G=(V, E)$ be a simple, undirected graph with vertex set $V$ and edge set $E$. For $x, y \in V$ we denote by $d(x, y)$ the distance between $x$ and $y$, which is the number of edges in a shortest $(x, y)$-path. The $r$ th power of a graph $G$, written as $G^{r}$, is a graph on the same vertex set such that two vertices are joined by an edge if and only if their distance in $G$ is at most $r$. For $r \geq 1$, let $C_{n}^{r}$ and $P_{n}^{r}$ denote the $r$ th power of a cycle and a path on $n$ vertices, respectively.

A $b$-coloring of a graph $G$ by $k$ colors is a proper vertex coloring such that each color class contains a vertex having neighbors in all other $k-1$ color classes. Such a vertex is called a color-dominating vertex. The $b$-chromatic number $\chi_{b}(G)$ is the maximum integer $k$ for which $G$ has a $b$-coloring by $k$ colors. The concept of $b$-colorings and the $b$-chromatic number were introduced by Irving and Manlove [5] in 1999 and have already been investigated in more than 50 papers (cf. [1]-[7]).

Since the problem to decide whether $\chi_{b}(G) \geq K$ for a given graph $G$ and an integer $K$ is $\mathcal{N P}$-hard in general $([5])$, we are interested in exact values on the $b$-chromatic number for special graphs or graph classes. In 2003, Effantin and Kheddouci [3] determined $\chi_{b}\left(P_{n}^{r}\right)$ for all values of $n$ and $r$. They also investigated powers of cycles $C_{n}^{r}$ and obtained the following result:

\footnotetext{
${ }^{\dagger}$ Email: kohleinformatik.htw-dresden.de.

1365-8050 @ 2013 Discrete Mathematics and Theoretical Computer Science (DMTCS), Nancy, France
} 
Theorem 1 (Effantin and Kheddouci, [3]) The b-chromatic number of $C_{n}^{r}$ for $r \geq 1$ is

$$
\begin{aligned}
\chi_{b}\left(C_{n}^{r}\right)= \begin{cases}n & \text { if } n \leq 2 r+1, \\
r+1 & \text { if } n=2 r+2, \\
r+1+\left\lfloor\frac{n-r-1}{3}\right\rfloor & \text { if } 3 r+1 \leq n \leq 4 r, \\
2 r+1 & \text { if } n \geq 4 r+1,\end{cases} \\
\text { and } \chi_{b}\left(C_{n}^{r}\right) \geq \min \left\{n-r-1, r+1+\left\lfloor\frac{n-r-1}{3}\right\rfloor\right\} \quad \text { if } 2 r+3 \leq n \leq 3 r .
\end{aligned}
$$

The determination of $\chi_{b}\left(C_{n}^{r}\right)$ for $2 r+3 \leq n \leq 3 r$ was posed as an open problem, but the authors conjectured that the lower bound in (2) is the exact value.

In the present paper, we complete Effantin and Kheddouci's work by determining $\chi_{b}\left(C_{n}^{r}\right)$ for the missing cases $2 r+3 \leq n \leq 3 r$. It turns out that the lower bound in 2 is indeed the exact value on $\chi_{b}\left(C_{n}^{r}\right)$ for $2 r+3+\frac{r-6}{4}<n \leq 3 r$, but not for $2 r+3 \leq n \leq 2 r+3+\frac{r-6}{4}$.

\section{Main Result}

Theorem 2 Let $C_{n}^{r}$ be the rth power of a cycle of order $n$ with $2 r+3 \leq n \leq 3 r$. Moreover, set $l:=n-2(r+1), R:=(r+1+3 l) \bmod (5 l)$, and $m:=\max \left\{\left\lfloor\frac{R-2 l}{3}\right\rfloor, 0\right\}$. Then, $\chi_{b}\left(C_{n}^{r}\right)=\frac{3 n-R}{5}+m$.

\subsection{Preliminaries}

In the following, we only consider powers of cycles $C_{n}^{r}$ for which $2 r+3 \leq n \leq 3 r$ is satisfied.

Let $V$ be the vertex set of $C_{n}^{r}$ and set $l:=n-2(r+1)$. Then, the complement of $C_{n}^{r}$ is an $(l+1)$-regular graph and $1 \leq l \leq r-2$. Moreover, $C_{n}^{r}$ has independence number 2 .

Let $c$ be a $b$-coloring of $C_{n}^{r}$ by $k:=\chi_{b}\left(C_{n}^{r}\right)$ colors and let $V_{1}, \ldots, V_{k}$ be the corresponding color classes such that $1 \leq\left|V_{1}\right| \leq\left|V_{2}\right| \leq \ldots \leq\left|V_{k}\right| \leq 2$. By $a$ we denote the number of color classes of cardinality 1 . Choose a color-dominating vertex $v_{i} \in V_{i}$ for $i=1, \ldots, k$. Let $A:=\left\{v_{1}, \ldots, v_{a}\right\}, B:=\left\{v_{a+1}, \ldots, v_{k}\right\}$, and $C:=\left\{w_{a+1}, \ldots, w_{k}\right\}$ where $w_{i}$ is the partner of $v_{i}$, i.e. the vertex in $V_{i} \backslash\left\{v_{i}\right\}$, for $i \in\{a+1, \ldots, k\}$. Vertices from the set $A, B$, and $C$ are called $A$-, $B$-, and $C$-vertices, respectively.

A vertex-row $X$ shall be a set of vertices from $V$ which are consecutive on the underlying cycle $C_{n}$. If $X$ only consists of (non-) $A$-vertices, then we call it a (non-) A-vertex-row (analogously for $B$ and $C$ ).

The neighborhood $N(x)$ of a vertex $x \in V$ is the set of neighbors of $x$. Moreover, let $\bar{N}(x):=$ $V \backslash(N(x) \cup\{x\})$ be the non-neighborhood of $x$ and for a set $V^{\prime} \subseteq V$ let $\bar{N}\left(V^{\prime}\right):=\bigcup_{x \in V^{\prime}} \bar{N}(x)$. Note that $|\bar{N}(X)|=l+|X|$ for every vertex-row $X$.

For a vertex $u \in V$ and a set $V^{\prime} \subseteq V$ let $d_{C_{n}}\left(u, V^{\prime}\right):=\min \left\{d_{C_{n}}(u, v) \mid v \in V^{\prime}\right\}$ where $d_{C_{n}}(u, v)$ denotes the distance of $u$ and $v$ in the underlying $C_{n}$.

We refer to Figure 1 for the following considerations.

Let $X$ be an $A$-vertex-row with $\alpha \geq 1$ vertices. We denote the two subgraphs induced by the vertex-rows between $X$ and $\bar{N}(X)$ in clockwise order and in anti-clockwise order (on the underlying $C_{n}$ ) by $G_{r}(X)$ and $G_{l}(X)$, respectively (compare left side of Figure 1). From above we know that $\bar{N}(X)$ contains exactly $l+\alpha$ vertices. Moreover, the set $\bar{N}(\bar{N}(X)) \backslash X$ has then exactly $2 l$ vertices, and by symmetry we obtain $\left|\bar{N}(\bar{N}(X)) \cap V\left(G_{l}(X)\right)\right|=\left|\bar{N}(\bar{N}(X)) \cap V\left(G_{r}(X)\right)\right|=l$. Since each vertex from $\bar{N}(X)$ is non-adjacent to at least one $A$-vertex (which corresponds to a color class of cardinality 1 ), it cannot be color-dominating. So, each vertex from $\bar{N}(X)$ is a $C$-vertex and therefore has a $B$-vertex as partner. Let $\bar{N}_{p}(X)$ be the set of partners of the vertices from $\bar{N}(X)$. Clearly, $\bar{N}_{p}(X) \subseteq \bar{N}(\bar{N}(X)) \backslash X$. 

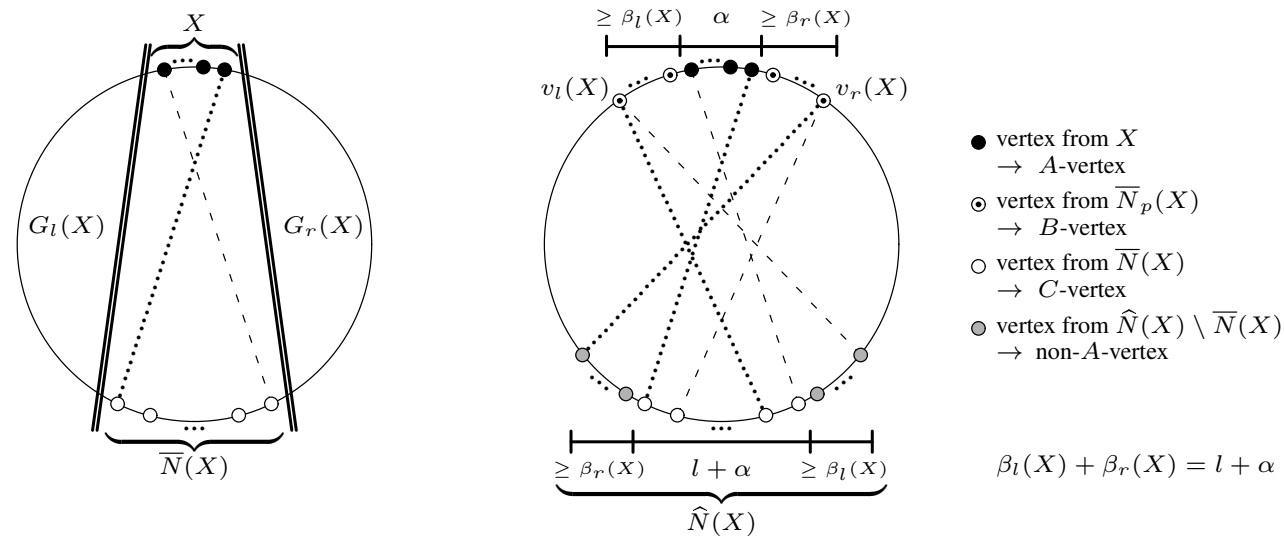

Fig. 1: Non-neighborhood $\bar{N}(X)$ and extended non-neighborhood $\widehat{N}(X)$ of an $A$-vertex-row $X$

Let $v_{l}(X)$ be the vertex from $\bar{N}_{p}(X) \cap V\left(G_{l}(X)\right)$ that have the largest distance to the set $X$ in $C_{n}$, i.e. $d_{C_{n}}\left(v_{l}(X), X\right)=\max _{v \in \bar{N}_{p}(X) \cap V\left(G_{l}(X)\right)} d_{C_{n}}(v, X)$. Moreover, set $\beta_{l}(X):=\left|\bar{N}_{p}(X) \cap V\left(G_{l}(X)\right)\right|$. Analogously, we define $v_{r}(X)$ and $\beta_{r}(X)$. From above we know that $\beta_{l}(X) \leq d_{C_{n}}\left(v_{l}(X), X\right) \leq$ $\left|\bar{N}(\bar{N}(X)) \cap V\left(G_{l}(X)\right)\right|=l$ and, analogously, $\beta_{r}(X) \leq d_{C_{n}}\left(v_{r}(X), X\right) \leq l$. Along with $\beta_{l}(X)+$ $\beta_{r}(X)=\left|\bar{N}_{p}(X)\right|=|\bar{N}(X)|=l+\alpha \geq l+1$, we further obtain $\beta_{l}(X), \beta_{r}(X) \geq 1$. This implies that both vertices $v_{l}(X)$ and $v_{r}(X)$ exist.

Since the partners of $v_{l}(X)$ and $v_{r}(X)$ are in $\bar{N}(X)$, we know that the sets $\bar{N}\left(v_{l}(X)\right)$ and $\bar{N}(X)$ as well as the sets $\bar{N}\left(v_{r}(X)\right)$ and $\bar{N}(X)$ intersect, and we can determine that $\left|\bar{N}\left(v_{l}(X)\right) \backslash \bar{N}(X)\right|=$ $d_{C_{n}}\left(v_{l}(X), X\right)$ and $\left|\bar{N}\left(v_{r}(X)\right) \backslash \bar{N}(X)\right|=d_{C_{n}}\left(v_{r}(X), X\right)$. Since each vertex from $\bar{N}\left(v_{l}(X)\right)$ is nonadjacent to the color-dominating vertex $v_{l}(X)$, it cannot be an $A$-vertex. The same holds for the vertices from $\bar{N}\left(v_{r}(X)\right)$. So, altogether, $\bar{N}\left(v_{l}(X)\right) \cup \bar{N}(X) \cup \bar{N}\left(v_{r}(X)\right)$ is a non- $A$-vertex-row with $l+\alpha+$ $d_{C_{n}}\left(v_{l}(X), X\right)+d_{C_{n}}\left(v_{r}(X), X\right) \geq l+\alpha+\beta_{l}(X)+\beta_{r}(X)=2(l+\alpha)$ vertices. We call this set the extended non-neighborhood of $X$ and denote it by $\widehat{N}(X)$. If $X$ consists of only one vertex $x$, then we just write $\widehat{N}(x)$ instead of $\widehat{N}(\{x\})$. The same we do with all previously defined notations.

Observation 1 Let $X_{1}, \ldots, X_{t}$ be a family oft $\geq 1$ disjoint $A$-vertex-rows (in clockwise order on the $C_{n}$ ) such that $\bar{N}\left(X_{i}\right) \cap \bar{N}\left(X_{i+1}\right) \neq \emptyset$ for $i \in\{1, \ldots, t-1\}$. Moreover, we assume the given A-vertex-rows to be maximal, i.e. each of it is delimited by non-A-vertices. Then, $\sum_{i=1}^{t}\left|X_{i}\right| \leq l$.

Proof: Let $Y_{i}$ be the non- $A$-vertex-row between $X_{i}$ and $X_{i+1}$ for $i \in\{1, \ldots, t-1\}$ and let $Y_{0}$ and $Y_{t}$ be the vertex-rows with $l$ vertices preceding $X_{1}$ and succeeding $X_{t}$, respectively. Moreover, set $\mathcal{X}:=$ $\bigcup_{i=1}^{t} X_{i}$ and $\mathcal{Y}:=\bigcup_{i=1}^{t-1} Y_{i}$. Note that $\mathcal{X} \cup \mathcal{Y}$ is a vertex-row and therefore satisfies $|\bar{N}(\mathcal{X} \cup \mathcal{Y})|=$ $l+|\mathcal{X} \cup \mathcal{Y}|$.

Since $\bar{N}\left(X_{i}\right)$ and $\bar{N}\left(X_{i+1}\right)$ intersect for each $i \in\{1, \ldots, t-1\}$, it follows that $\bar{N}\left(Y_{i}\right) \subset\left(\bar{N}\left(X_{i}\right) \cup\right.$ $\left.\bar{N}\left(X_{i+1}\right)\right)$ and therefore $\bar{N}(\mathcal{Y}) \subset \bar{N}(\mathcal{X})$. Moreover, it implies that $\bar{N}(\mathcal{X})$ is a $C$-vertex-row. For its cardinality we obtain: 


$$
\begin{aligned}
\left|\bar{N}_{p}(\mathcal{X})\right| & =|\bar{N}(\mathcal{X})|=|\bar{N}(\mathcal{X}) \cup \bar{N}(\mathcal{Y})|=|\bar{N}(\mathcal{X} \cup \mathcal{Y})|=l+|\mathcal{X} \cup \mathcal{Y}| \\
& =l+\left|\bigcup_{i=1}^{t} X_{i} \cup \bigcup_{i=1}^{t-1} Y_{i}\right|=l+\left|\bigcup_{i=1}^{t} X_{i}\right|+\left|\bigcup_{i=1}^{t-1} Y_{i}\right|=l+\sum_{i=1}^{t}\left|X_{i}\right|+\sum_{i=1}^{t-1}\left|Y_{i}\right| .
\end{aligned}
$$

Moreover, we can easily check that $\bar{N}(\bar{N}(\mathcal{X}))=\bigcup_{i=1}^{t} X_{i} \cup \bigcup_{i=0}^{t} Y_{i}=\mathcal{X} \cup \bigcup_{i=0}^{t} Y_{i}$. Since $\bar{N}_{p}(\mathcal{X}) \subseteq$ $\bar{N}(\bar{N}(\mathcal{X})) \backslash \mathcal{X}$, we then obtain:

$$
\left|\bar{N}_{p}(\mathcal{X})\right| \leq|\bar{N}(\bar{N}(\mathcal{X})) \backslash \mathcal{X}|=\left|\bigcup_{i=0}^{t} Y_{i}\right| \leq \sum_{i=0}^{t}\left|Y_{i}\right|=2 l+\sum_{i=1}^{t-1}\left|Y_{i}\right| .
$$

So Inequalities 3 , and (4) yield $\sum_{i=1}^{t}\left|X_{i}\right| \leq l$.

Note that for $t=1$, the previous observation yields that every $A$-vertex-row contains at most $l$ vertices.

\subsection{Proof of Theorem 2}

Recall that $k=\chi_{b}\left(C_{n}^{r}\right), a=|A|$, and $l=n-2(r+1)$. Since the $b$-coloring by $k$ colors partitions the vertex set into $a$ color classes of cardinality 1 and $k-a$ color classes of cardinality 2, we obtain $n=a+2(k-a)$ and, thus, $a=2 k-n=2 k-(l+2 r+2)=2(k-r-1)-l$. So, $a, l$, and $n$ have the same parity. Moreover, according to Inequality $\left\{2, k \geq \min \left\{n-(r+1), r+1+\left\lfloor\frac{n-(r+1)}{3}\right\rfloor\right\}=\right.$ $\min \left\{l+r+1, r+1+\left\lfloor\frac{l+r+1}{3}\right\rfloor\right\}=r+1+\min \left\{l,\left\lfloor\frac{l+r+1}{3}\right\rfloor\right\}$ and therefore

$$
a+l=2 k-n+l=2 k-2(r+1) \geq 2 \min \left\{l,\left\lfloor\frac{l+r+1}{3}\right\rfloor\right\} .
$$

Let $Q$ and $R$ be the quotient and the remainder of the integer division $r+1+3 l$ by $5 l$, i.e. $r+1+3 l=$ $Q \cdot 5 l+R$, where $Q \geq 0$ and $0 \leq R<5 l$. Note that then $Q=\left\lfloor\frac{r+1+3 l}{5 l}\right\rfloor, R=(r+1+3 l) \bmod (5 l)$, and $n=l+2(r+1)=(2 Q-1) 5 l+2 R$. Moreover, set $m:=\max \left\{\left\lfloor\frac{R-2 l}{3}\right\rfloor, 0\right\}$.

Case 1. $l+3 \leq r+1<2 l$, i.e. $\frac{5(r+1)}{2}<n \leq 3 r$.

At first we notice that this case is only possible for $l \geq 4$. Moreover, by (5) it follows that

$$
a+l \geq 2\left\lfloor\frac{l+r+1}{3}\right\rfloor \geq \frac{2(l+r-1)}{3}=\frac{2 r-(l+2)}{3}+l \geq \frac{l+2}{3}+l \geq 2+l .
$$

Hence, there exist at least two $A$-vertices. Choose two vertices $u_{1}, u_{2} \in A$ of maximum distance in $C_{n}$. W.l.o.g. $u_{1}$ and $u_{2}$ are ordered in such a way, that a shortest $\left(u_{1}, u_{2}\right)$-path in $C_{n}$ runs anti-clockwise. Then there are two cases to consider depending on the positions of $u_{2}$ and $v_{l}\left(u_{1}\right)$ (compare Figure 2). From the preliminaries and the premise of the case, we know that the extended non-neighborhood of $u_{1}$ has cardinality $\left|\widehat{N}\left(u_{1}\right)\right| \geq 2(l+1) \geq r+4$. So, since $\widehat{N}\left(u_{1}\right)$ is a vertex-row of at least $r+4$ vertices, but the maximal vertex-rows induced by $N\left(u_{2}\right)$ have only $r$ vertices, we deduce that $u_{2}$ cannot be adjacent to all vertices in $\widehat{N}\left(u_{1}\right)$, and so $\widehat{N}\left(u_{1}\right)$ and $\bar{N}\left(u_{2}\right)$ intersect. Hence, $\widehat{N}\left(u_{1}\right) \cup \bar{N}\left(u_{2}\right)$ is a non- $A$-vertex-row. 

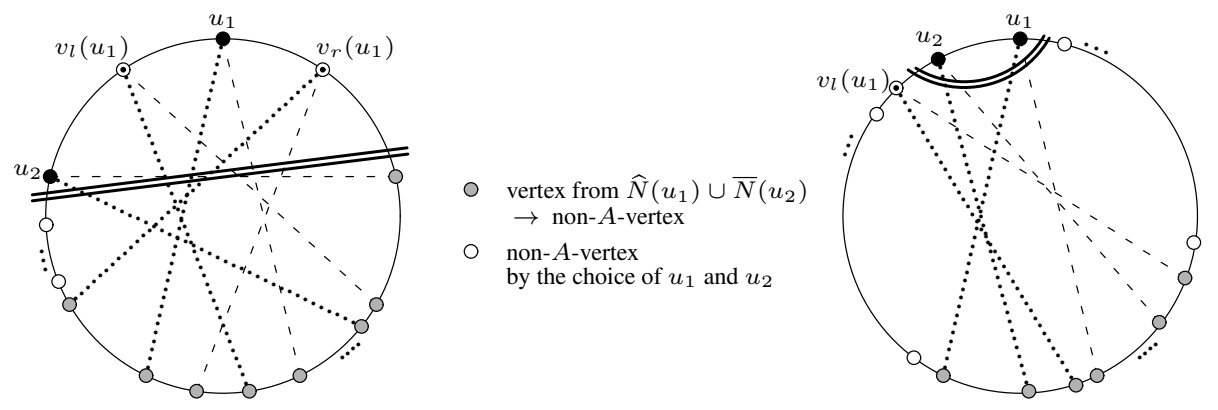

Fig. 2: Extended non-neighborhood $\widehat{N}\left(u_{1}\right)$ and non-neighborhood $\bar{N}\left(u_{2}\right)$ intersect

Subcase 1.1. $u_{2}$ and $v_{l}\left(u_{1}\right)$ are in clockwise order (see left side of Figure 2).

Since $\widehat{N}\left(u_{1}\right) \cup \bar{N}\left(u_{2}\right)$ is a non- $A$-vertex-row and by the choice of $u_{1}$ and $u_{2}$ as two $A$-vertices of maximum distance, there is no $A$-vertex in $V\left(G_{l}\left(u_{2}\right)\right) \cup \bar{N}\left(u_{2}\right)$ (below the double-line). The remaining vertex set $V\left(G_{r}\left(u_{2}\right)\right) \cup\left\{u_{2}\right\}$ (above the double-line) induces a vertex-row with exactly $r+1$ vertices. This vertexrow has to contain all $a A$-vertices and all $l+1 B$-vertices from $\bar{N}_{p}\left(u_{1}\right)$. Hence, $r+1 \geq a+l+1$. This and the premise of the case yield:

$$
a+l \leq r=\frac{(r+2)+2(r+1)-4}{3} \leq \frac{2 l+2(r+1)-4}{3} \leq 2\left\lfloor\frac{l+r+1}{3}\right\rfloor .
$$

Along with Inequality 6 , we obtain $a+l=2\left\lfloor\frac{l+r+1}{3}\right\rfloor$.

Subcase 1.2. $u_{2}$ and $v_{l}\left(u_{1}\right)$ are in anti-clockwise order (see right side of Figure 2).

By the choice of $u_{1}$ and $u_{2}$, there is no $A$-vertex in $V\left(G_{l}\left(u_{2}\right)\right) \cup \bar{N}\left(u_{2}\right) \cup V\left(G_{r}\left(u_{1}\right)\right) \cup \bar{N}\left(u_{1}\right)$ (below the double-curve). Hence, the remaining vertex set $\left(V\left(G_{r}\left(u_{2}\right)\right) \cap V\left(G_{l}\left(u_{1}\right)\right)\right) \cup\left\{u_{1}, u_{2}\right\}$ (above the double-curve) that induces a vertex-row with $1+d_{C_{n}}\left(u_{1}, u_{2}\right)$ vertices has to contain all $a A$-vertices. Since $d_{C_{n}}\left(u_{1}, u_{2}\right) \leq d_{C_{n}}\left(u_{1}, v_{l}\left(u_{1}\right)\right)-1 \leq l-1$, the non-neighborhoods $\bar{N}\left(u_{1}\right)$ and $\bar{N}\left(u_{2}\right)$ intersect. This implies that $\bar{N}(A)=\bar{N}\left(u_{1}\right) \cup \bar{N}\left(u_{2}\right)$ and therefore $|\bar{N}(A)|=l+1+d_{C_{n}}\left(u_{1}, u_{2}\right) \geq l+a$. Since $\bar{N}(A) \subseteq C, \bar{N}_{p}(A) \subseteq B$, and $|\bar{N}(A)|=\left|\bar{N}_{p}(A)\right|$, we deduce that $n=|A|+|B|+|C| \geq$ $a+\left|\bar{N}_{p}(A)\right|+|\bar{N}(A)|=a+2 \cdot|\bar{N}(A)| \geq a+2(l+a)=3 a+2 l$. Along with $n=l+2(r+1)$ we then obtain $a+l \leq\left\lfloor\frac{2(l+r+1)}{3}\right\rfloor$. This and Inequality 6 yield

$$
2\left\lfloor\frac{l+r+1}{3}\right\rfloor \leq a+l \leq\left\lfloor\frac{2(l+r+1)}{3}\right\rfloor,
$$

and since $a$ and $l$ have the same parity implying that $a+l$ is even, we conclude that $a+l=2\left\lfloor\frac{l+(r+1)}{3}\right\rfloor$.

Hence, in both subcases we obtain $a+l=2\left\lfloor\frac{l+r+1}{3}\right\rfloor$ which implies that $k=r+1+\left\lfloor\frac{l+r+1}{3}\right\rfloor$. Moreover, since $r+1+3 l<5 l$ by the premise of the case, it follows that $R=r+1+3 l$ and $m=\left\lfloor\frac{r+1+l}{3}\right\rfloor$. Therefore, $k=r+1+\left\lfloor\frac{l+r+1}{3}\right\rfloor=r+1+m=\frac{3(l+2(r+1))-(r+1+3 l)}{5}+m=\frac{3 n-R}{5}+m$.

Case 2. $r+1 \geq 2 l \geq 2$, i.e. $2 r+3 \leq n \leq \frac{5(r+1)}{2}$.

The premise yields $r+1+3 l=Q \cdot 5 l+R \geq 5 l$ and therefore $Q \geq 1$. 


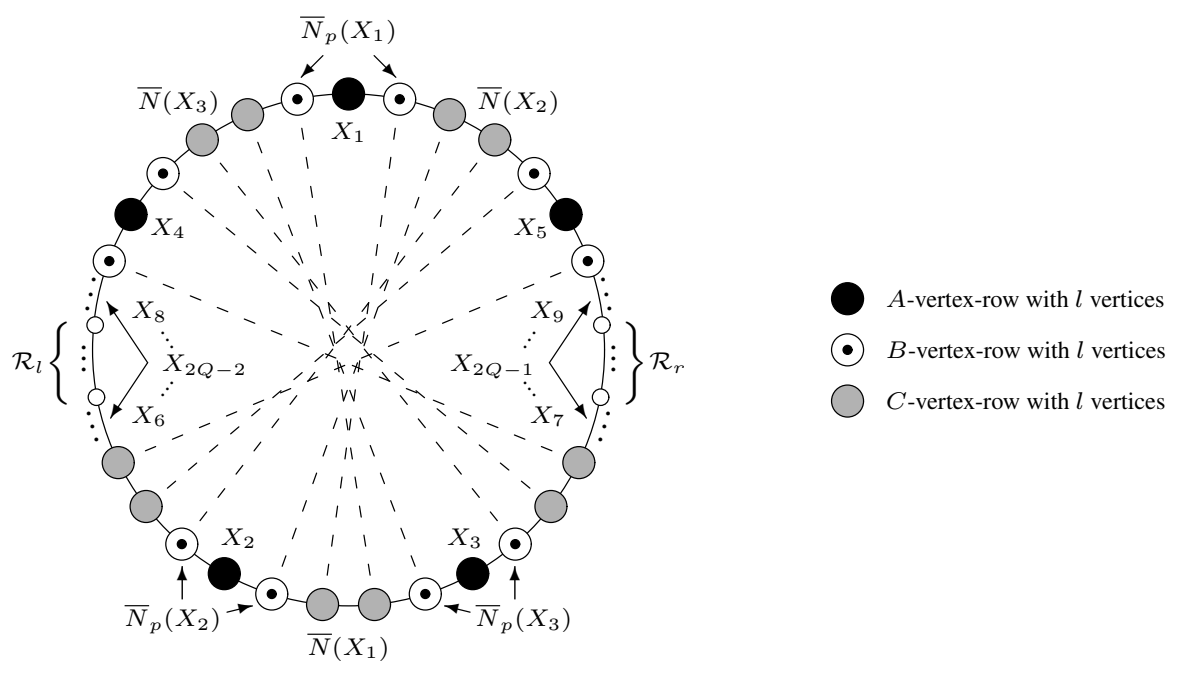

Fig. 3: Partition of $V$ into $2 Q-1$ bundles of $5 l$ vertices each and $2 R$ remaining vertices

[" $\geq$ "] At first we prove that $k \geq(2 Q-1) 3 l+R+m=\frac{3 n-R}{5}+m$. In order to do this we construct a $b$-coloring of $C_{n}^{r}$ by $k^{\prime}:=(2 Q-1) 3 l+R+m$ colors.

Since $n=(2 Q-1) 5 l+2 R$, we can partition the vertex set $V$ into $2 Q-1$ bundles $\mathcal{B}_{1}, \ldots, \mathcal{B}_{2 Q-1}$ of $5 l$ vertices each and $2 R$ remaining vertices. For $i \in\{1, \ldots, 2 Q-1\}$, the bundle $\mathcal{B}_{i}$ shall consist of a vertex-row $X_{i}$ with $l$ black vertices, the non-neighborhood $\bar{N}\left(X_{i}\right)$ with $2 l$ grey vertices, and the set $\bar{N}_{p}\left(X_{i}\right):=\bar{N}\left(\bar{N}\left(X_{i}\right)\right) \backslash X_{i}$ with $2 l$ black-white vertices, such that the sets $X_{1}, \ldots, X_{2 Q-1}$ are positioned on the cycle $C_{n}$ as described in Figure 3 In order to do this, we start with an arbitrary vertex-row with $l$ vertices as $X_{1}$ (on top in the picture). Then the positions of $\bar{N}\left(X_{1}\right)$ and $\bar{N}_{p}\left(X_{1}\right)$ are already specified. After this, we position $X_{2}$ (bottom left) and $X_{3}$ (bottom right) leaving out $l$ vertices on each side of $\bar{N}\left(X_{1}\right)$. Then the positions of $\bar{N}\left(X_{2}\right)$ and $\bar{N}_{p}\left(X_{2}\right)$ as well as $\bar{N}\left(X_{3}\right)$ and $\bar{N}_{p}\left(X_{3}\right)$ are already specified. If $Q \geq 3$, then we proceed by positioning $X_{4}$ (top left) and $X_{5}$ (top right) leaving out $l$ vertices on the left side of $\bar{N}\left(X_{3}\right)$ and on the right side of $\bar{N}\left(X_{2}\right)$, respectively. Then also the positions of $\bar{N}\left(X_{4}\right)$ and $\bar{N}_{p}\left(X_{4}\right)$ as well as $\bar{N}\left(X_{5}\right)$ and $\bar{N}_{p}\left(X_{5}\right)$ are specified. If $Q \geq 4$, then we position the remaining sets $X_{6}, \ldots, X_{2 Q-1}$ in the same manner as we positioned $X_{2}-X_{5}$ using always the order bottom left, bottom right, top left, top right. The $2 R$ remaining vertices induce two vertex-rows with $R$ vertices each which we denote by $\mathcal{R}_{l}$ and $\mathcal{R}_{r}$.

Now we color the $l$ vertices from $X_{1}$ in clockwise order by colors $1, \ldots, l$. Then we color the $2 l$ vertices from $\bar{N}\left(X_{1}\right)$ in clockwise order by colors $l+1, \ldots, 3 l$, and the same we do for the $2 l$ vertices from $\bar{N}\left(\bar{N}\left(X_{1}\right)\right) \backslash X_{1}=\bar{N}_{p}\left(X_{1}\right)$. Now all vertices from $\mathcal{B}_{1}$ are colored. Next we color the vertices from $\mathcal{B}_{2}$ in an analogous way by colors $3 l+1, \ldots, 6 l$, and we continue until $\mathcal{B}_{2 Q-1}$ whose vertices we color by colors $(2 Q-2) 3 l+1, \ldots,(2 Q-1) 3 l$. This coloring procedure yields a coloring of $(2 Q-1) 5 l=n-2 R$ vertices by $(2 Q-1) 3 l$ colors (compare the left side of Figure 5 for the case $l=Q=2$ ). For the coloring of the vertices from $\mathcal{R}_{l} \cup \mathcal{R}_{r}$ we have to distinguish the following two cases: 




Fig. 4: Partition of the remaining $2 R$ vertices for the case $m>0$

Subcase 2.1. $0 \leq R \leq 2(l+1)$.

This implies that $m=0$. We color the vertices from $\mathcal{R}_{l}$ by colors $(2 Q-1) 3 l+1, \ldots,(2 Q-1) 3 l+R$ in clockwise order. The same we do with the vertices from $\mathcal{R}_{r}$. Altogether, we obtain a coloring $c_{b}$ of $C_{n}^{r}$ by $(2 Q-1) 3 l+R=k^{\prime}$ colors.

Subcase 2.2. $2(l+1)<R<5 l$.

Here, $m>0$. We partition $\mathcal{R}_{l} \cup \mathcal{R}_{r}$ as depicted in Figure 4 into two bundles $\mathcal{B}_{2 Q}, \mathcal{B}_{2 Q+1}$ of $2 l+3 m$ vertices each and two vertex-rows $\mathcal{R}_{l}^{\prime}$ and $\mathcal{R}_{r}^{\prime}$ of $R-2 l-3 m$ vertices each. For $i \in\{2 Q, 2 Q+1\}$, the bundle $\mathcal{B}_{i}$ shall consist of a vertex-row $X_{i}$ with $m$ black vertices, the non-neighborhood $\bar{N}\left(X_{i}\right)$ with $l+m$ grey vertices, and $\bar{N}_{p}\left(X_{i}\right)$ which is a subset of $l+m$ black-white vertices from the set $\bar{N}\left(\bar{N}\left(X_{i}\right)\right) \backslash X_{i}$.

Now we color the vertices from $X_{2 Q}$ in clockwise order by colors $(2 Q-1) 3 l+1, \ldots,(2 Q-1) 3 l+m$. Then we color the vertices from $\bar{N}\left(X_{2 Q}\right)$ as well as from $\bar{N}_{p}\left(X_{2 Q}\right)$ in clockwise order by colors $(2 Q-$ 1) $3 l+m+1, \ldots,(2 Q-1) 3 l+2 m+l$. Analogously, we color the vertices from $X_{2 Q+1}, \bar{N}\left(X_{2 Q+1}\right)$, and $\bar{N}_{p}\left(X_{2 Q+1}\right)$ by colors $(2 Q-1) 3 l+2 m+l+1, \ldots,(2 Q-1) 3 l+4 m+2 l$. Finally, we color the vertices from $\mathcal{R}_{l}^{\prime}$ as well as from $\mathcal{R}_{r}^{\prime}$ in clockwise order by colors $(2 Q-1) 3 l+4 m+2 l+1, \ldots,(2 Q-$ 1) $3 l+4 m+2 l+(R-2 l-3 m)=(2 Q-1) 3 l+R+m$ (compare the right side of Figure 5 for the case $l=Q=2, m=1)$. Altogether, this yields a coloring $c_{b}$ of $C_{n}^{r}$ by $(2 Q-1) 3 l+R+m=k^{\prime}$ colors.

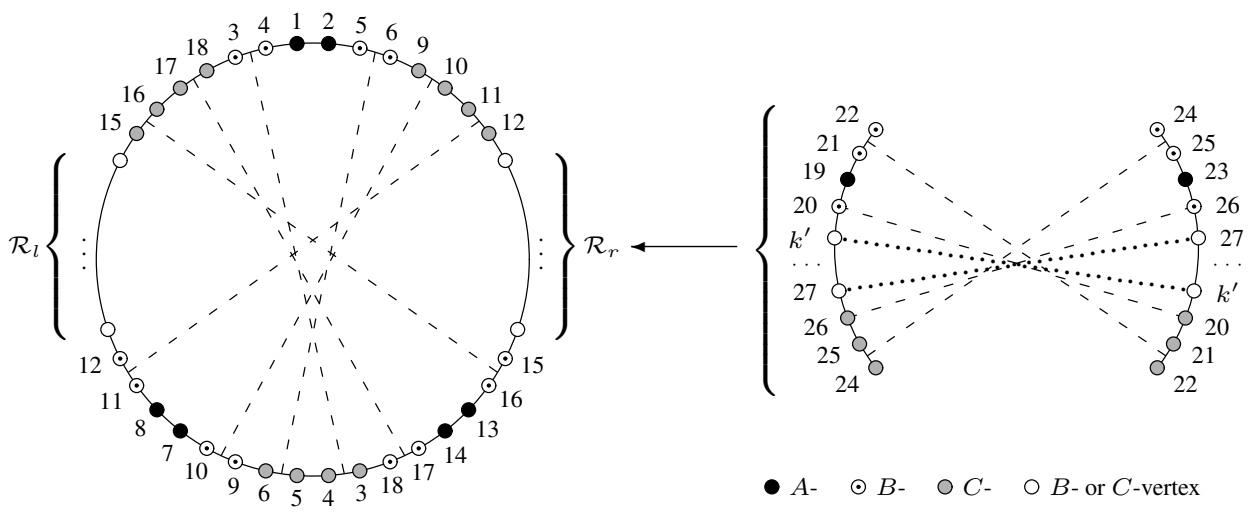

Fig. 5: $b$-coloring by $k^{\prime}=(2 Q-1) 3 l+R+m$ colors (here for $l=Q=2, m=1$ ) 
It remains to prove that $c_{b}$ is a $b$-coloring for both subcases. Consider the Figures $3 \mid 5$. The black vertices correspond to the color classes of cardinality 1 in the coloring $c_{b}$. Moreover, each color class of cardinality 2 consists of a black-white and a grey vertex. Note that in Subcase 2.1 (resp. 2.2) we assume the vertices in $R_{l}$ (resp. $R_{l}^{\prime}$ ) to be black-white and the vertices in $R_{r}$ (resp. $R_{r}^{\prime}$ ) to be grey.

Because $C_{n}^{r}$ has independence number 2, a vertex $x$ from a color class $V_{j}$ has at least one neighbor in each color class $V_{g}$ of cardinality 2 for $j \neq g$. Hence, $x$ is color-dominating if and only if $x$ is adjacent to each vertex in a color class $V_{h}$ of cardinality 1 for $j \neq h$, i.e. if $x$ is adjacent to all (other) black vertices.

Along with the fact that the black vertices are the vertices from $\bigcup_{i=1}^{s} X_{i}(s:=2 Q-1$ in Subcase 2.1 and $s:=2 Q+1$ in Subcase 2.2) this implies that $x$ is color-dominating if and only if $x \notin \bar{N}\left(\bigcup_{i=1}^{s} X_{i}\right)=$ $\bigcup_{i=1}^{s} \bar{N}\left(X_{i}\right)$. Since all vertices from $\bigcup_{i=1}^{s} \bar{N}\left(X_{i}\right)$ are grey, we deduce that the black and the black-white vertices are color-dominating and correspond to the $A$ - and the $B$-vertices, respectively. The grey vertices are then the $C$-vertices. Moreover, since every color class contains either a black or a black-white vertex it follows that every color class has a color-dominating vertex. Hence, $c_{b}$ is a proper $b$-coloring of $C_{n}^{r}$.

For the number $k^{\prime}$ of colors used in $c_{b}$ we obtain:

$$
\begin{aligned}
k^{\prime} & =(2 Q-1) 3 l+R+m=r+1+Q l+m=\frac{5(r+1)+(r+1+3 l-R)}{5}+m \\
& =\frac{3(l+2(r+1))-R}{5}+m=\frac{3 n-R}{5}+m .
\end{aligned}
$$

This implies that $k \geq k^{\prime}$ and because of $a=2 k-n$ we further deduce that $a \geq 2 k^{\prime}-n=2(r+1+$ $Q l+m)-(l+2(r+1))=(2 Q-1) l+2 m$.

[" $\leq$ "] Now we prove that $k \leq r+1+Q l+m=\frac{3 n-R}{5}+m$.

Consider the $b$-coloring $c$ of $C_{n}^{r}$ by $k$ colors and suppose that $k>k^{\prime}$. This is equivalent to $a>(2 Q-$ 1) $l+2 m$. Since $a$ and $l$ have the same parity, it suffices to consider $a \geq(2 Q-1) l+2(m+1)$. Choose an arbitrary $A$-vertex-row $X$ with $\alpha, 1 \leq \alpha \leq l$, vertices. We refer to Figure 1 for the following considerations.

Let $\beta_{l}(X)$ and $\beta_{r}(X)$ be defined as before. In the following, we abbreviate these values by $\beta_{l}$ and $\beta_{r}$. Moreover, let $a_{l}:=\left|A \cap V\left(G_{l}(X)\right)\right|, b_{l}:=\left|\left(B \backslash \bar{N}_{p}(X)\right) \cap V\left(G_{l}(X)\right)\right|$, and $c_{l}:=\left|C \cap V\left(G_{l}(X)\right)\right|$. Analogously, we define $a_{r}, b_{r}$, and $c_{r}$ for the subgraph $G_{r}(X)$. Since $G_{l}(X)$ and $G_{r}(X)$ have the same order, we deduce that $\left|V\left(G_{l}(X)\right)\right|=\beta_{l}+a_{l}+b_{l}+c_{l}=\left|V\left(G_{r}(X)\right)\right|=\beta_{r}+a_{r}+b_{r}+c_{r}$. Every vertex from the set $\left(B \backslash \bar{N}_{p}(X)\right) \cap V\left(G_{l}(X)\right)$ has a partner in $C \cap V\left(G_{r}(X)\right)$, and vice versa. Hence, $b_{l}=c_{r}$. Analogously, we obtain $b_{r}=c_{l}$. Moreover, $\beta_{l}+\beta_{r}=\left|\bar{N}_{p}(X)\right|=|\bar{N}(X)|=l+\alpha$ and $a=\alpha+a_{l}+a_{r}$. Therefore, $\beta_{l}+a_{l}=\beta_{r}+a_{r}=\left(l+\alpha-\beta_{l}\right)+\left(a-\alpha-a_{l}\right)$ and altogether, $2\left(\beta_{l}+a_{l}\right)=2\left(\beta_{r}+a_{r}\right)=l+a$. By the assumption $a \geq(2 Q-1) l+2(m+1)$ we obtain $2\left(\beta_{l}+a_{l}\right)=2\left(\beta_{r}+a_{r}\right)=l+a \geq 2 Q l+2(m+1)$ and thus, $\beta_{l}+a_{l}=\beta_{r}+a_{r} \geq Q l+m+1$. Moreover, since $1 \leq \beta_{l}, \beta_{r} \leq l$ (compare the preliminaries), we deduce that $Q l+m+1 \leq \beta_{l}+a_{l} \leq l+a_{l}$ which yields $a_{l} \geq(Q-1) l+m+1$. Analogously, we obtain $a_{r} \geq(Q-1) l+m+1$.

Let $X_{1}, \ldots, X_{q^{\prime}}$ be the family of all maximal $A$-vertex-rows in $V\left(G_{l}(X)\right)$ (in clockwise order). For $i \in$ $\left\{1, \ldots, q^{\prime}-1\right\}$ merge the two sets $X_{i}$ and $X_{i+1}$ if $\bar{N}\left(X_{i}\right) \cap \bar{N}\left(X_{i+1}\right) \neq \emptyset$. Continue doing this until the remaining sets, say $\mathcal{X}_{1}, \ldots, \mathcal{X}_{q}$ (in clockwise order), satisfy $\bar{N}\left(\mathcal{X}_{i}\right) \cap \bar{N}\left(\mathcal{X}_{i+1}\right)=\emptyset$ for $i \in\{1, \ldots, q-1\}$. Set $\alpha_{i}:=\left|\mathcal{X}_{i}\right|$ for $i \in\{1, \ldots, q\}$. Obviously, $\sum_{i=1}^{q} \alpha_{i}=a_{l}$. Moreover, from Observation 1$]$ we know that $\alpha_{i} \leq l$ for $i \in\{1, \ldots, q\}$. Hence, $q l \geq \sum_{i=1}^{q} \alpha_{i}=a_{l} \geq(Q-1) l+m+1 \geq(Q-1) l+1$ and therefore $q \geq Q$. 
Since every vertex from $\bigcup_{i=1}^{q} \bar{N}\left(\mathcal{X}_{i}\right)$ is a $C$-vertex from $V\left(G_{r}(X)\right)$ we further deduce that $b_{l}=c_{r} \geq$ $\left|\bigcup_{i=1}^{q} \bar{N}\left(\mathcal{X}_{i}\right)\right|=\sum_{i=1}^{q}\left|\bar{N}\left(\mathcal{X}_{i}\right)\right| \geq \sum_{i=1}^{q}\left(l+\alpha_{i}\right)=a_{l}+q l \geq a_{l}+Q l$. Analogously, we can show that $b_{r}=c_{l} \geq a_{r}+Q l$. For the number of vertices $n$ we then obtain:

$$
\begin{aligned}
n & =|X|+|\bar{N}(X)|+\left|\bar{N}_{p}(X)\right|+a_{l}+a_{r}+b_{l}+b_{r}+c_{l}+c_{r} \\
& =\alpha+2(l+\alpha)+\left(a_{l}+a_{r}\right)+2\left(b_{l}+b_{r}\right)=a+2(l+\alpha)+2\left(b_{l}+b_{r}\right) \\
& \geq a+2(l+\alpha)+2\left(a_{r}+a_{l}+2 Q l\right)=3 a+2 l+4 Q l \\
& \geq 3(2 Q-1) l+6(m+1)+2 l+4 Q l=10 Q l-l+6(m+1)
\end{aligned}
$$

Moreover, since $n=(2 Q-1) 5 l+2 R$, Inequality 99 yields $R \geq 2 l+3(m+1)$ and therefore $\left\lfloor\frac{R-2 l}{3}\right\rfloor \geq$ $m+1$. This is a contradiction to $m=\max \left\{\left\lfloor\frac{R-2 l}{3}\right\rfloor, 0\right\}$.

Therefore, $k \leq k^{\prime}$, and altogether $k=k^{\prime}=\frac{3 n-R}{5}+m$.

\section{Conclusion}

In [3], Effantin and Kheddouci conjectured that $\chi_{b}\left(C_{n}^{r}\right)=\min \left\{n-(r+1), r+1+\left\lfloor\frac{n-(r+1)}{3}\right\rfloor\right\}$ for $2 r+3 \leq n \leq 3 r$. The following observation shows that this conjecture does not hold in general.

Observation 2 Let $C_{n}^{r}$ be the rth power of a cycle of order $n$ with $2 r+3 \leq n \leq 3 r$, and set $L:=$ $\min \left\{n-(r+1), r+1+\left\lfloor\frac{n-(r+1)}{3}\right\rfloor\right\}$. Then, $\chi_{b}\left(C_{n}^{r}\right)=L$ if $n>\frac{9 r+6}{4}$ and $\chi_{b}\left(C_{n}^{r}\right)>L$ if $n \leq \frac{9 r+6}{4}$.

Proof: Recall that $l=n-2(r+1), R=(r+1+3 l) \bmod (5 l), Q=\frac{r+1+3 l-R}{5 l}$, and $m=$ $\max \left\{\left\lfloor\frac{R-2 l}{3}\right\rfloor, 0\right\}$. So we obtain $L=r+1+\min \left\{l,\left\lfloor\frac{r+1+l}{3}\right\rfloor\right\}$.

Case 1. $l+3 \leq r+1<2 l$, i.e. $\frac{5(r+1)}{2}<n \leq 3 r$.

Then, $L=r+1+\left\lfloor\frac{r+1+l}{3}\right\rfloor$. Moreover, we obtain $r+1+3 l<5 l$ which implies that $R=r+1+3 l$ and $m=\left\lfloor\frac{R-2 l}{3}\right\rfloor=\left\lfloor\frac{r+1+l}{3}\right\rfloor$. Thus, Theorem 2 yields $\chi_{b}\left(C_{n}^{r}\right)=\frac{3 n-R}{5}+m=\frac{3(l+2(r+1))-(r+1+3 l)}{5}+$ $\left\lfloor\frac{r+1+l}{3}\right\rfloor=r+1+\left\lfloor\frac{r+1+l}{3}\right\rfloor=L$.

Case 2. $2 l \leq r+1<4 l+3$, i.e. $\frac{9 r+6}{4}<n \leq \frac{5(r+1)}{2}$.

Then, $L=r+1+l$. Furthermore, it follows that $5 l \leq r+1+3 l<7 l+3=5 l+(2 l+3)$ and therefore $Q=1$ and $0 \leq R=r+1-2 l<2 l+3$. This yields $m=0$. Due to Theorem 2, we then obtain $\chi_{b}\left(C_{n}^{r}\right)=\frac{3 n-R}{5}=\frac{3(l+2(r+1))-(r+1-2 l)}{5}=r+1+l=L$.

Case 3. $r+1 \geq 4 l+3 \geq 7$, i.e. $2 r+3 \leq n \leq \frac{9 r+6}{4}$.

Then, $L=r+1+l$. Moreover, we deduce that $r+1+3 l \geq 7 l+3=5 l+(2 l+3)$ which yields $Q \geq 1$. According to Theorem 2, $\chi_{b}\left(C_{n}^{r}\right)=\frac{3 n-R}{5}+m=r+1+l+\frac{r+1-2 l-R}{5}+m=L+\frac{r+1-2 l-R}{5}+m$ (note that $\frac{r+1-2 l-R}{5}$ is an integer). In order to prove $\chi_{b}\left(C_{n}^{r}\right)>L$, we have to show that $\frac{r+1-2 l-R}{5}+m \geq 1$.

If $m=0$, then $R \leq 2 l+2$ and therefore $\frac{r+1-2 l-R}{5}=\left\lceil\frac{r+1-2 l-R}{5}\right\rceil \geq\left\lceil\frac{r+1-4 l-2}{5}\right\rceil \geq\left\lceil\frac{1}{5}\right\rceil=1$.

If $m>0$, then $\frac{r+1-2 l-R}{5}+m=\frac{r+1-2 l-(r+1+3 l-5 l Q)}{5}+m=(Q-1) l+m \geq 0+m \geq 1$.

\section{Acknowledgements}

The author would like to thank the referees for helpful comments and suggestions. 


\section{References}

[1] M. Alkhateeb, A. Kohl, Upper bounds on the b-chromatic number and results for restricted graph classes, Discussiones Mathematicae Graph Theory 31 (4) (2011), 709-735.

[2] M. Blidia, F. Maffray, Z. Zemir, On b-colorings in regular graphs, Discrete Appl. Math. 157 (2009), 1787-1793.

[3] B. Effantin, H. Kheddouci, The b-chromatic number of some power graphs, DMTCS 6 (2003), 045054.

[4] C.T. Hoang, M. Kouider, On the b-dominating coloring of graphs, Discrete Appl. Math. 152 (2005), 176-186.

[5] R.W. Irving, D.F. Manlove, The b-chromatic number of a graph, Discrete Appl. Math. 91 (1-3) (1999), 127-141.

[6] M. Kouider, M. Mahéo, Some bounds for the b-chromatic number of a graph, Disc. Math. 256 (2002), 267-277.

[7] J. Kratochvíl, Zs. Tuza, M. Voigt, On the b-chromatic number of graphs, WG 2002, LNCS 2573 (2002), 310-320. 